\title{
Numerical Study of the Action of Convection on the Volume and Length of the Flammable Zone Formed by Hydrogen Emissions from the Vent Masts Installed on an International Ship
}

\author{
Hyeonsu Nam ${ }^{1}$, Gun Woo Kim ${ }^{2}$, Hyunyong Lee ${ }^{3}$, , Byung Chul Choi ${ }^{4}$, Gilltae Roh ${ }^{3}$ and Youngseung Na ${ }^{1, * *(1)}$ \\ 1 Department of Mechanical and Information Engineering, Seoul 02504, Korea; ehf121ro@uos.ac.kr \\ 2 Offshore Platform Research Division, Korea Research Institute of Ships \& Ocean Engineering, \\ Daejeon 34103, Korea; gwkim@kriso.re.kr \\ 3 R\&D Center, Korean Register, Busan 46762, Korea; leehy@krs.co.kr (H.L.); gtroh@krs.co.kr (G.R.) \\ 4 Division of Mechanical and Automotive Engineering, Hoseo University, Asan 31499, Korea; \\ choibc@hoseo.edu \\ * Correspondence: ysna@uos.ac.kr; Tel.: +82-02-6490-2395
}

\section{check for} updates

Citation: Nam, H.; Kim, G.W.; Lee, H.; Choi, B.C.; Roh, G.; Na, Y. Numerical Study of the Action of Convection on the Volume and Length of the Flammable Zone Formed by Hydrogen Emissions from the Vent Masts Installed on an International Ship. J. Mar. Sci. Eng. 2021, 9, 1348. https://doi.org/ $10.3390 /$ jmse 9121348

Academic Editor: Tie Li

Received: 26 October 2021

Accepted: 26 November 2021

Published: 30 November 2021

Publisher's Note: MDPI stays neutral with regard to jurisdictional claims in published maps and institutional affiliations.

Copyright: (C) 2021 by the authors Licensee MDPI, Basel, Switzerland. This article is an open access article distributed under the terms and conditions of the Creative Commons Attribution (CC BY) license (https:// creativecommons.org/licenses/by/ $4.0 /)$.

\begin{abstract}
International ships carrying liquefied fuel are strongly recommended to install vent masts to control the pressure of cargo tanks in the event of an emergency. However, the gas emitted from a vent mast may be hazardous for the crew of the ship. In the present study, the volume and length of the flammable zone (FZ) created by the emitted gas above the ship was examined. Various scenarios comprising four parameters, namely, relative wind speed, arrangement of vent masts, combination of emissions among four vent masts, and direction of emission from the vent-mast outlet were considered. The results showed that the convection acts on the volume and length of an FZ. The volume of an FZ increases when there is a reduction in convection reaching the FZ and when strong convection brings hydrogen from a nearby FZ. The length of the FZ is also related to convection. An FZ is elongated if the center of a vortex is located inside the FZ, because this vortex traps hydrogen inside the FZ. The length of an FZ decreases if the center of the vortex is located outside the FZ, as such a vortex brings more fresh air into the FZ.
\end{abstract}

Keywords: international shipping; liquefied hydrogen; flammability limit; vent mast; ventilation

\section{Introduction}

The threat of climate change is a significant global issue, according to the Paris Agreement. Most of the countries bound by this agreement must make significant efforts to reduce greenhouse gas (GHG) emissions as soon as possible [1], since large GHG emissions accelerate global warming. In this context, the International Maritime Organization (IMO) has agreed to limit GHG emissions, with the goal of completely eliminating GHGs produced by international shipping by the end of the century [2].

GHG emissions from the use of fossil fuels are unavoidable since GHG contains not only carbon dioxide but also methane, the principal component of natural gas, which is increasingly being used as a substitute for traditional fossil fuels [3,4]. The IMO's goal includes the use of carbon-free fuels such as hydrogen gas in international ships. As a result, they are working to update previous safety requirements, such as the IGF code [5], so that they can be applied to ships that use hydrogen as a fuel [6].

New Energy and Industrial Technology Development Organization (NEDO) also introduced the supply chain of hydrogen [7]. According to the $\mathrm{CO}_{2}$-free Hydrogen Energy Supply-chain Technology Research Association (HyStra), the hydrogen supply chain requires the development of a liquefied hydrogen gas transporter [8]. A study on reliquefying systems evaluated the boil-off gas from liquefied hydrogen cargo tanks in these circumstances [9]. It assumed a natural boil-off rate of approximately $0.3 \%$ per day. 
The International Code of the Construction and Equipment of Ships Carrying Liquefied Gases in Bulk (IGC Code) is a safety regulation for the international shipping of liquefied fuel [10]. IMO mandates the installation of ventilation systems on the liquefied fuel gas carrier by IGC code to prevent any emergency, such as gas leakage. The last components of the ventilation system are the vent masts, which are positioned on the ship's deck. This vent mast allows a boiling gas to escape into the atmosphere. This mechanism is required to keep the cargo tank's pressure within the IGC code's authorized range; the highest permissible pressure for a membrane-type tank is $0.025 \mathrm{MPa}$ [10]. Another type of vent mast was developed to deal with gas leaking inside a tank or machine room. This type of vent mast was examined by Roh et al. [11] and Kim et al. [12] for a gas leakage inside a tank or machine room. The flammable zone (FZ) formation outside the room should also be considered because the IGC code addresses the threat of the FZ formed by ventilated gas for the crew. In the design factor of a vent mast, it recommends that the vent mast should be taller than one-third of the width of the ship, or $6 \mathrm{~m}$, and that the vent mast should depart from the steering room at least a width of the vessel or $25 \mathrm{~m}$ [10].

Doh et al. [13] analyzed the dispersion characteristics of the vented gas with a static swirl mixer on the outlet of a single vent mast, which only has an upward-facing open exit. Their research took into account scenarios in which the required gas discharge rate is expelled through the vent mast during an emergency. Blaylock et al. [14] used wind speed to examine the creation of the FZ from a single vent mast. They discovered that the wind has a considerable impact on the shape of that area. The zone is twisted toward the flow direction when the wind blows against the released hydrogen gas, and a pair of revolving vortexes form, swiftly dissipating the hydrogen gas. Kang [15] examined how wind speed affected the scattered concentration of boil-off gas from a single vent mast. This boil-off rate is not the same as the gas discharge rate stated by the IGC code [10]. Hansen [16] studied the shape of the FZ for a single vent mast under $2 \mathrm{~m} \mathrm{~s}^{-1}$ wind speed according to the air temperature. Cao et al. [17] investigated the FZ in relation to the height of a single vent mast and found that the higher the vent mast's peak, the safer the crew is. Cornwell et al. [18] explored the length of the FZ from a single vent mast based on the flow rate of gas from the vent mast.

All of the prior research was carried out for a single vent mast. However, there are more design considerations for many vent masts, such as the placement of vent masts and the combination of emissions. The length and volume of the FZ may be changed by the combinations of a variety of vent masts. As a result, this article is concerned with a large number of vent masts. This study looked at four different ventilation scenarios, each with its own set of parameters: relative wind speed, arrangement of vent masts, the combination of emission patterns selected from among the four vent masts, and ventilation direction of the vent-mast outlet. It uses a numerical method of commercial software STAR-CCM+ version 13.06.012.R8 to solve the computational fluid dynamics (CFD).

\section{Methods}

\subsection{Modeling}

The ship model has four tanks of 40,000 $\mathrm{m}^{3}$ each, representing a membrane-type liquefied gas carrier of about 50,000 dead weight tonnage (DWT) [19]. The part of the ship below the design load water line (DLWL) is not considered. The four vent masts are set on the top of each tank. It maintains an adequate distance for safety of the inhabitants. In compliance with the safety design rules in the IGC code, the height of each vent mast is $16 \mathrm{~m}$, which is one-third of the width of the ship. The detailed geometry of the ship is shown in Figure 1. The atmospheric space is adequate, as shown in Figure 2. The properties of gas and air are summarized in Table 1 . The pressure $\left(P_{h}\right)$ of the computational domain is estimated from Equation (1), based on the height $(h)$ from the DLWL.

$$
P_{h}=P_{a t m}-\rho_{a t m} g h
$$


where atmospheric pressure $\left(P_{a t m}\right)$ is $101,325 \mathrm{~Pa}$; $\rho_{\text {humid }}$ is the density of the atmospheric air as calculated from Equation (2); and $g$ is acceleration due to gravity $\left(9.81 \mathrm{~m} / \mathrm{s}^{2}\right)$.

$$
\rho_{\text {atm }}=x_{\text {vapor }} \rho_{\text {vapor }}+x_{\text {dryair }} \rho_{\text {dryair }}
$$

where $x_{\text {vapor }}$ and $x_{\text {dryair }}$ are the volume fractions of vapor and dry air, respectively.

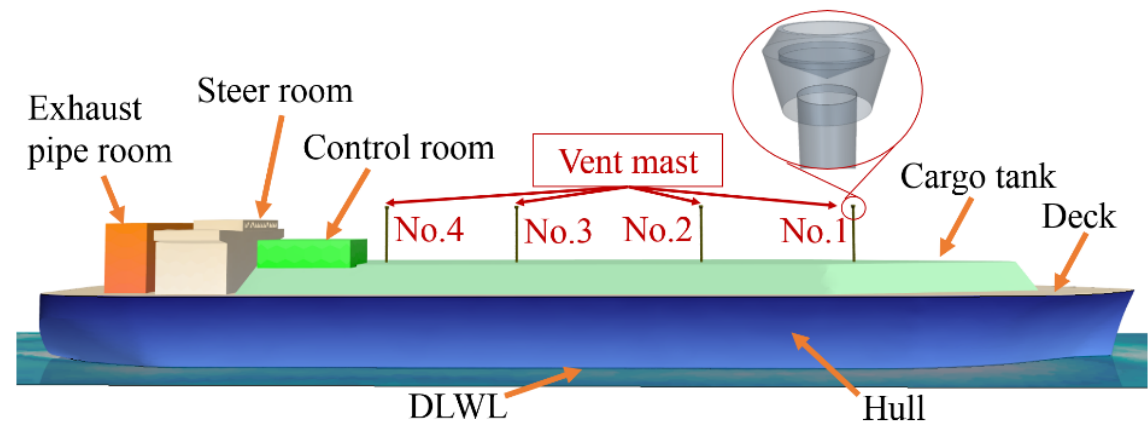

(a)

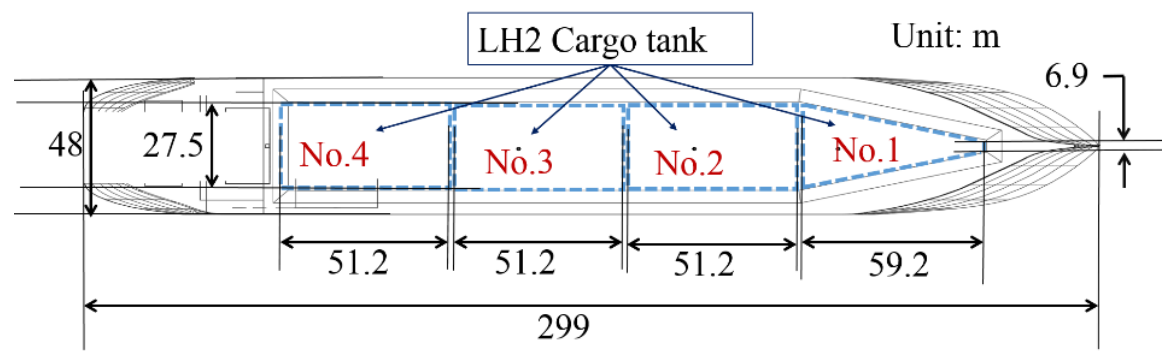

(b)

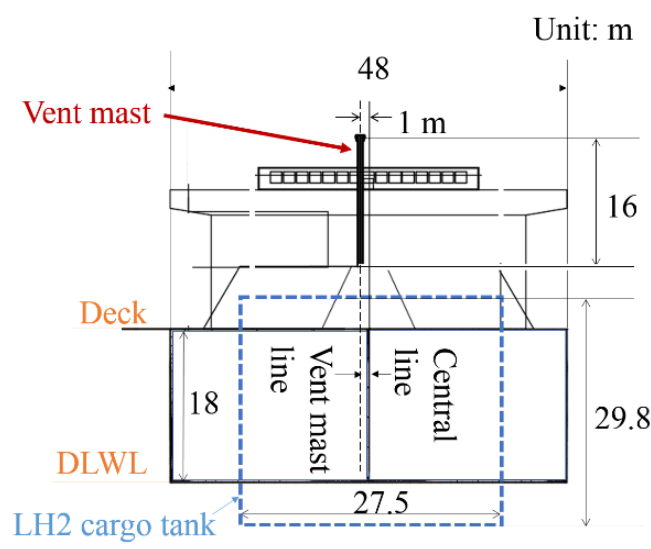

(c)

Figure 1. Design of the liquefied hydrogen (LH2) carrier equipped with four vent masts and four cargo tanks: (a) Three dimensional (3D) model (b) top view; and (c) front view.

\subsection{CFD Solvers}

In the present simulation, temperature solvers were not used. Instead, a segregated flow solver was used. The gas density was assumed constant. The Reynolds-averaged Navier-Stokes and $k-\epsilon$ turbulence models were used to solve the fluid mechanics. Fuel emissions were supposed to be ceaseless enough to form a stable FZ at a steady relative wind speed. Atmospheric air was assumed to consist of dry air and vapor. The gravity force had a buoyancy effect on the hydrogen in the air. 


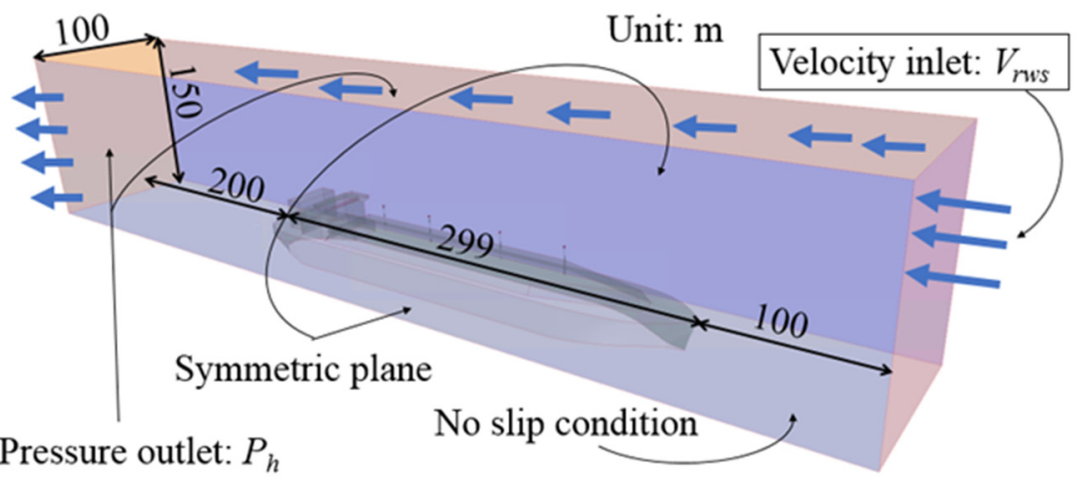

(a)

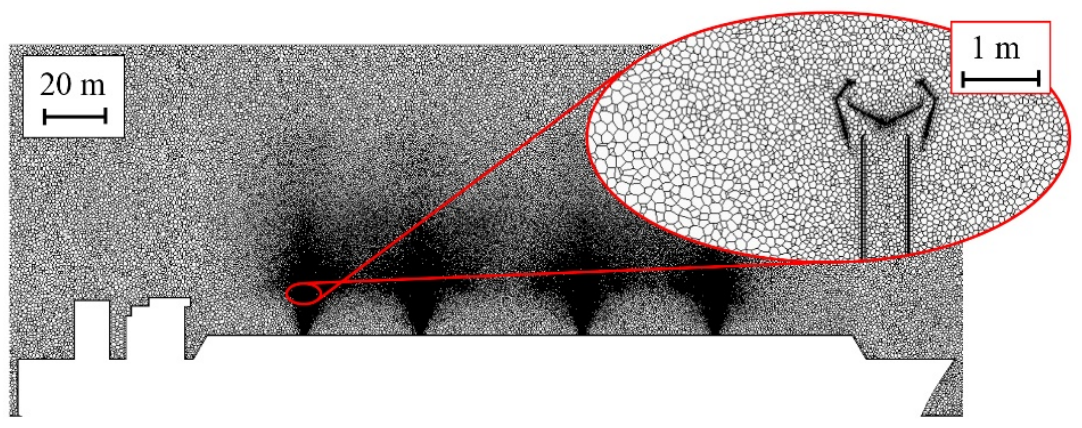

(b)

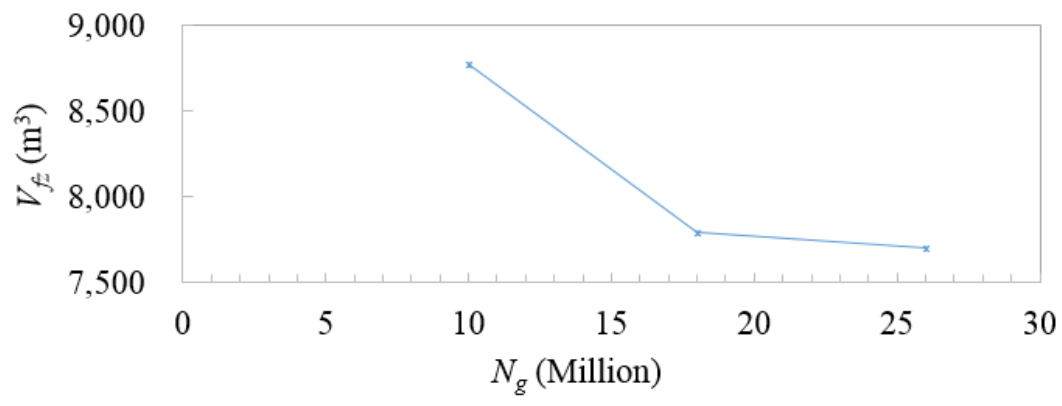

(c)

Figure 2. Computational domain and grid: (a) Boundary conditions for the atmospheric space above DLWL around the ship. (b) Composing mesh for the domain. (c) Grid dependency of the volume of $\mathrm{FZ}\left(V_{f z}\right)$ based on the grid numbers $\left(N_{g}\right)$.

Table 1. Gas properties of the computational domain.

\begin{tabular}{cccc}
\hline Parameter & Value & Reference & Unit \\
\hline$\rho_{\text {air }}$ & 1.198 & {$[20]$} & $\mathrm{kg} / \mathrm{m}^{3}$ \\
$\rho_{H 2}$ & 0.084 & {$[20]$} & $\mathrm{kg} / \mathrm{m}^{3}$ \\
$\rho_{\text {vapor }}$ & 0.59531 & & $\mathrm{~kg} / \mathrm{m}^{3}$ \\
$\mu_{\text {air }}$ & $1.80 \times 10^{-5}$ & {$[21]$} & $\mathrm{Pa} \cdot \mathrm{s}$ \\
$\mu_{H 2}$ & $8.39 \times 10^{-6}$ & {$[22]$} & $\mathrm{Pa} \cdot \mathrm{s}$ \\
$\mu_{\text {vapor }}$ & $9.73 \times 10^{-6}$ & {$[23]$} & $\mathrm{Pa} \cdot \mathrm{s}$ \\
$R H$ & 64 & & $\%$ \\
$x_{\text {dryair }}$ & 0.990639 & {$[24]$} & \\
$x_{\text {vapor }}$ & 0.009361 & {$[24]$} & \\
\hline
\end{tabular}




\subsection{Estimation with Assumptions}

Table 2 lists the variables considered for estimating the minimum required discharge flow rate of hydrogen as defined in the IGC code [10]. The temperature at the relieving conditions was assumed as $53.15 \mathrm{~K}$. The size of the LH2 tanks was estimated to be fit for the ship capacity, as shown in Figure 1. The discharging rate of gas from the $i$ th tank was estimated from Equation (3). The gas factor $G$ and the constant coefficient $D$ were defined as per Equations (4) and (5), respectively. The mass flow rate of gas through each vent mast of the tank was estimated from Equation (6), where $m_{i}$ is the minimum required mass flow rate of gas from the $i$ th tank.

$$
\begin{gathered}
Q_{i}=F G A_{i}^{0.82} \\
G=\frac{12.4}{L D} \sqrt{\frac{Z T}{M_{H 2}}} \\
D=\sqrt{k_{H 2}\left(\frac{2}{k_{H 2}+1}\right)^{\frac{k_{H 2}+1}{k_{H 2}-1}}} \\
m_{H 2, i}=\rho_{H 2} Q_{i}
\end{gathered}
$$

Table 2. Parameters to estimate the minimum required discharge flow rate of hydrogen from each vent mast.

\begin{tabular}{cccc}
\hline Parameter & Value & Reference & Unit \\
\hline$F$ & 0.1 & {$[10]$} & \\
$A_{1}$ & 6534 & & $\mathrm{~m}^{2}$ \\
$A_{2}$ & 7500 & {$[22]$} & $\mathrm{m}^{2}$ \\
$L$ & 445.65 & {$[10]$} & $\mathrm{kJ} / \mathrm{kg}$ \\
$Z$ & 1 & {$[10]$} & $\mathrm{K}$ \\
$T_{\text {rel }}$ & 53.15 & {$[25]$} & $\mathrm{kg} / \mathrm{kmol}$ \\
$M_{H 2}$ & 2.016 & {$[22]$} & \\
$k_{H 2}$ & 1.383 & & $\mathrm{~kg} / \mathrm{m}^{3}$ \\
$\rho_{H 2}$ & 0.084 & {$[26]$} & $\mathrm{Pa}$ \\
$P_{a t m}$ & 101,325 & &
\end{tabular}

\subsection{Parameters}

The following four parameters were used in the numerical study on the volume and length of the FZ.

1. Relative wind speed.

2. Arrangement of the vent masts.

3. Combination of emissions selected from among the four vent masts.

4. Direction of emissions from the vent-mast outlet.

The relative wind speeds considered were 10,20 , and $30 \mathrm{~m} / \mathrm{s}$. These speeds assume that the wind blows on directly against the voyage direction of the ship at 0,10 , and $20 \mathrm{~m} / \mathrm{s}$, while the ship goes forward as a typical speed of $10 \mathrm{~m} / \mathrm{s}$ [19]. A fresh gale corresponds to a wind speed of $20 \mathrm{~m} / \mathrm{s}$ [27].

Four types of arrangement of vent masts were considered (Figure 3). The reference arrangement is shown in Figure 3a. As shown in Figure 3c, the 12-34 arrangement shows differentiated position of the vent masts in which the second vent mast is transposed $15 \mathrm{~m}$ forward and the third vent mast is $15 \mathrm{~m}$ backward. In the 1-23-4 arrangement, the second and third vent masts are transposed $15 \mathrm{~m}$ each to bring them close, as shown in Figure $3 \mathrm{~b}$. In the zigzag arrangement, the first and third vent masts are shifted each by $2 \mathrm{~m}$ toward the left side of the ship (Figure 3d). 


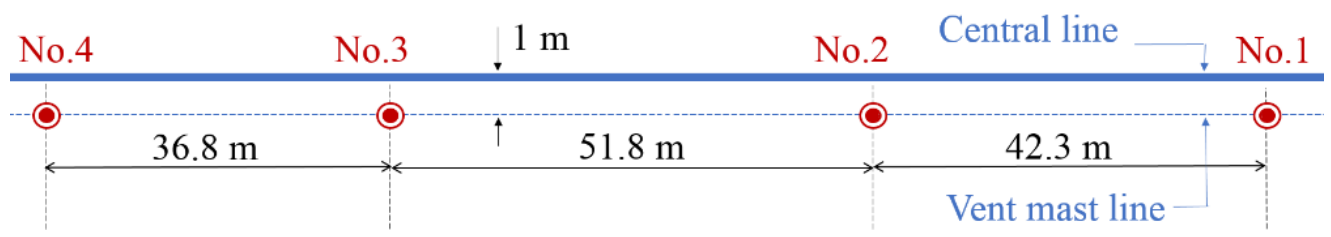

(a)

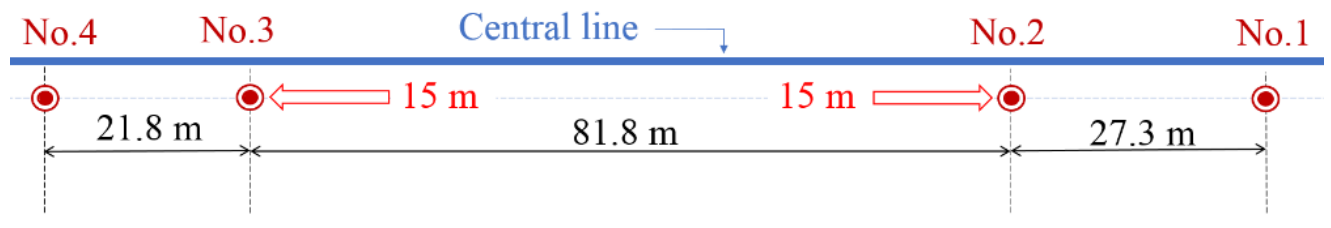

(b)

No.4 No.3 $\quad$ No.2 $\quad$ Central line $\longrightarrow$ No.1

(c)

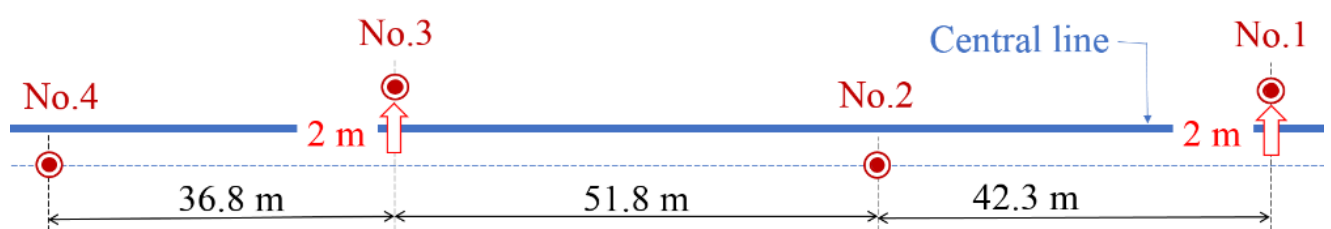

(d)

Figure 3. Four kinds of arrangements of the vent masts: (a) Reference (b) 1-23-4, (c) 12--34, and (d) zigzag.

Emissions from four vent masts can yield several possible combinations. Each scenario consists of a combination of emissions from four vent masts. The volumes of the FZs for these combinations were examined to identify how multiple emissions affect the volume and length of an FZ. The relative volume ratio of an FZ is defined as per Equation (7).

$$
R_{v f z}=\frac{V_{f z}}{V_{f z, 1}}
$$

where $V_{f z}$ is the total volume of the $\mathrm{FZ}$, and $V_{f z, 1}$ is the volume of the $\mathrm{FZ}$ when hydrogen is emitted from the first vent mast alone. The criteria for the FZ are on the volume fraction of hydrogen. This classification is based on the ClassNK group guideline [20] that introduces the criterion of standard flammability limit (FL) on the basis of hydrogen volume fraction.

Finally, three types of vent-mast outlets were designed to determine the effect of direction of emissions. The original design of vent mast outlet emits hydrogen into two directions, namely, upward and downward, as shown in Figure 4a, following the work of Kang [15]. The other two kinds of vent-mast outlet emit hydrogen either only downward or upward, as shown in Figure $4 b, c$, respectively. In all the cases, the vent-mast pipe diameter is set as $0.6 \mathrm{~m}$. 


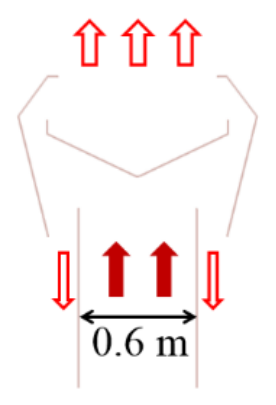

(a)

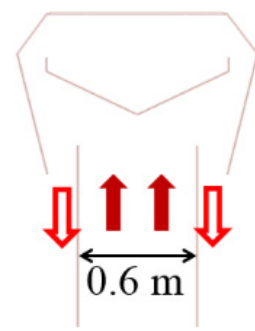

(b)

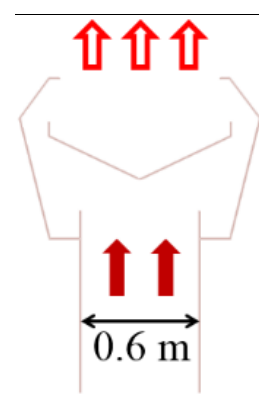

(c)

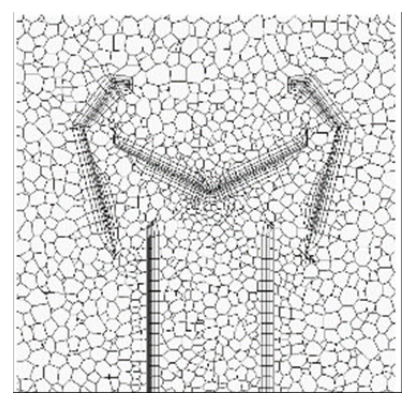

(d)

Figure 4. Three vent-mast outlet directions: (a) Upward and downward, (b) downward, (c) upward, and (d) grid around a vent-mast outlet.

It has been chosen for grid number, from a grid dependency test, on a volume of the $\mathrm{FZ}$ of a representative scenario. This scenario is the reference arrangement with a relative wind speed of $10 \mathrm{~m} / \mathrm{s}$. All the vent masts emit hydrogen from the outlet in upward and downward directions. It is less than $1.19 \%$ in a differential rate of volume of an FZ and is the ratio of the difference between the volumes obtained from two grid numbers of 26 and 18 million. Therefore, the number of grids for the computational domain in all other scenarios was chosen as 18 million.

The mass flow rate of hydrogen for each vent mast was determined from Equation (6). It is $2.37 \mathrm{~kg} / \mathrm{s}$ for the first vent mast and $2.65 \mathrm{~kg} / \mathrm{s}$ for the other three vent masts. These correspond to volume flow rates of $28.16 \mathrm{~m}^{3} / \mathrm{s}$ and $31.54 \mathrm{~m}^{3} / \mathrm{s}$, respectively, under the conditions listed in Table 2.

The FZ is sliced at $5 \mathrm{~m}$ intervals to inspect the FZ more closely at the cross sections. These sections begin from the center of the first vent-mast outlet and follow the drifting direction of hydrogen. The contour lines and vectors are drawn on these sections. These contour lines are distinguished by the ranges of volume fractions of hydrogen, as listed in Table 3. These ranges contain not only a standard volume fraction of the lower flammability limit (LFL), but also nearby fractions because the flame can extend up to the region of the lower volume fraction of $0.5 \%$, and the ignition of a hydrogen jet is restrained at the higher volume fraction range of $8-10 \%$ [28]. Hydrogen does not explode if the fraction is above $75 \%$ of the volume fraction - this limit is known as the upper flammability limit (UFL). In the present study, the FZ is defined in the range of volume fractions between the LFL and UFL.

Table 3. Colors assigned for each range of volume fraction of hydrogen.

\begin{tabular}{ccc}
\hline Name of Contour Line & Range of Volume Fraction (\%) & Color \\
\hline $12.5 \%$ of LFL & $0.5-1.0$ & Gray \\
LFL & $4.0-4.5$ & Red \\
$200 \%$ of LFL & $8.0-10.0$ & Brass \\
\hline
\end{tabular}

\section{Results and Discussion}

Figure 5a shows the contour lines on a cross section to denote the FL. Two types of lines are used as the criterion for a cross section. The first type is a vent-mast line shown in Figure 3a; it is used for the cross section shown in Figures 5, 6 and 10a,b. The second type is a vertical line (as marked in Figure 10a) perpendicular to the drifting direction. The vertical line is a criterion for the cross sections shown in Figures 10c,d and 13. 


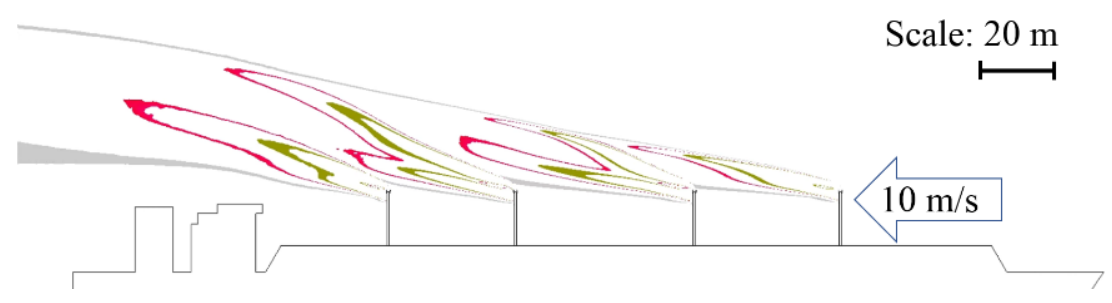

(a)

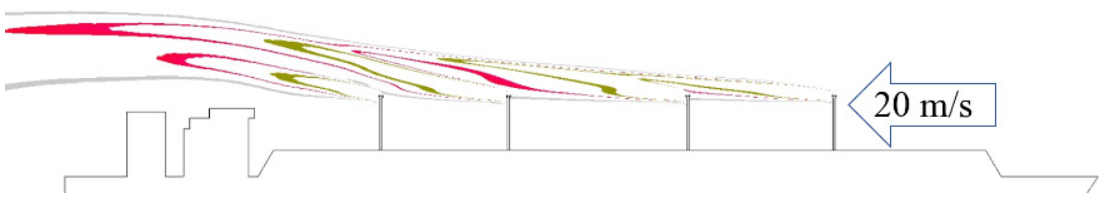

(b)

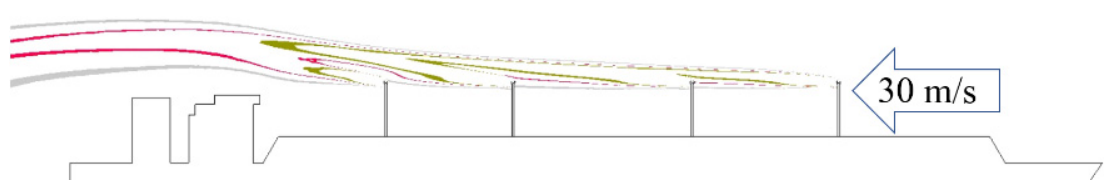

(c)

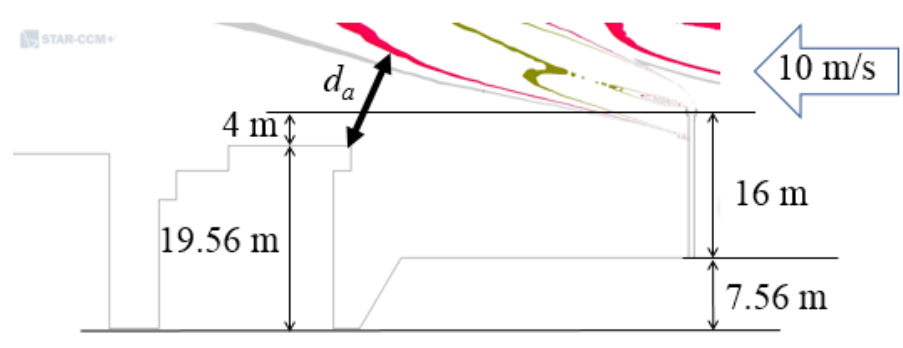

(d)

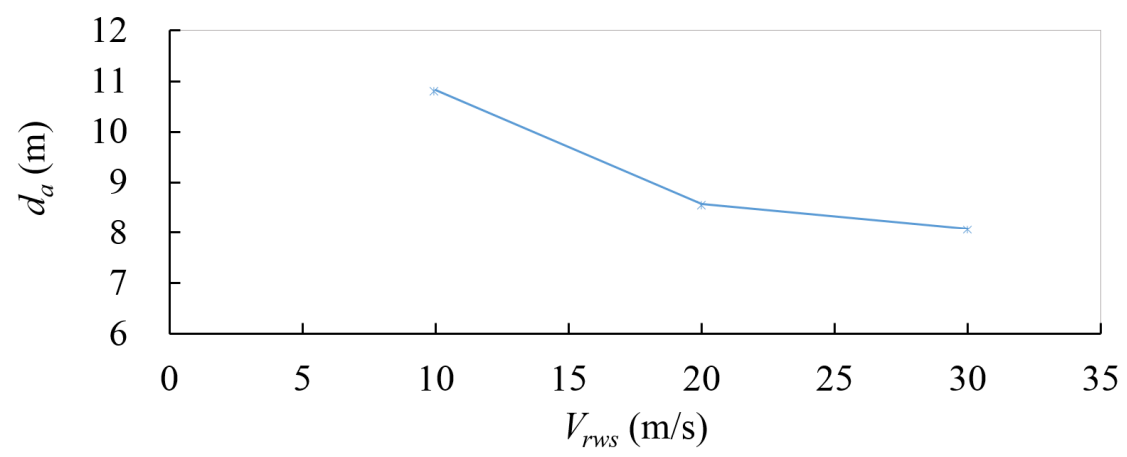

(e)

Figure 5. Contour lines of the flammability limits (FL) based on the relative wind speed ( $\left.V_{\text {rws }}\right)$ : Around the whole ship at a relative wind speed of (a) $10 \mathrm{~m} / \mathrm{s}$; (b) $20 \mathrm{~m} / \mathrm{s}$; (c) $30 \mathrm{~m} / \mathrm{s}$; (d) near the inhabitable area at $10 \mathrm{~m} / \mathrm{s}$; and (e) approximate minimum distances $\left(d_{a}\right)$ of FZ from the steer room.

\subsection{Relative Wind Speed}

In Figure 5, the FZ at each relative wind speed is shown. The minimum distance of the FZ from the steer room was measured. The distances were $8.7,9.2$, and $11.5 \mathrm{~m}$ at relative wind speeds of 30,20, and $10 \mathrm{~m} / \mathrm{s}$, respectively. Thus, the distance from the steer room decreases as the relative wind speed increases, as shown in Figure 5e. 


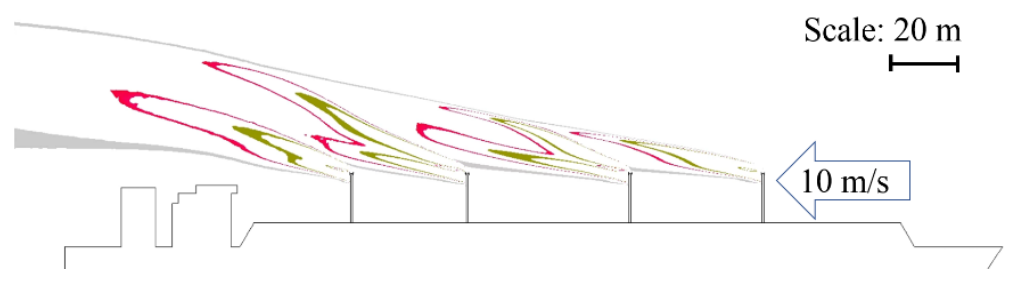

(a)

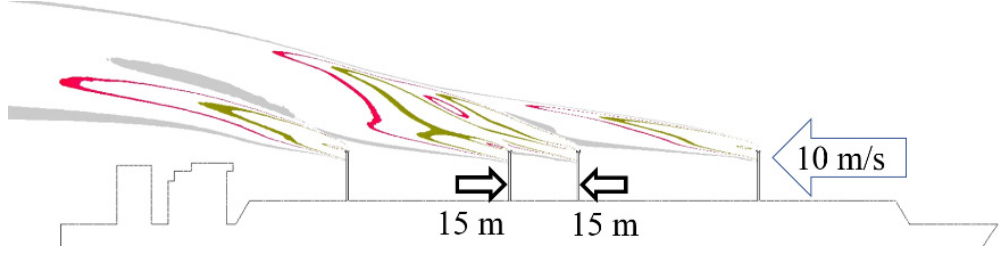

(b)

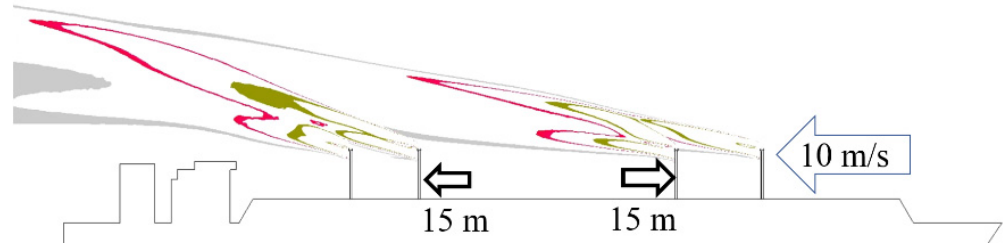

(c)

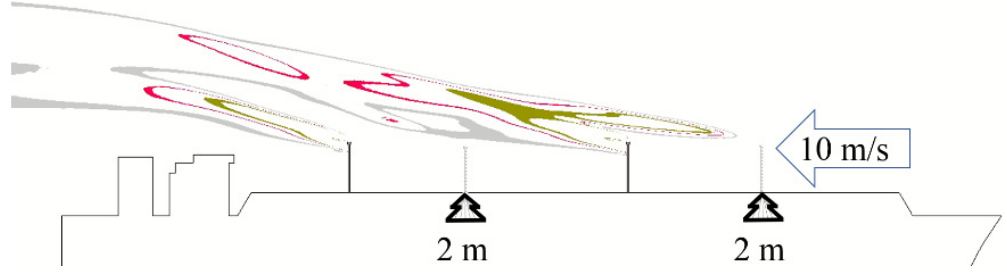

(d)

Figure 6. Contour lines of the FL on the cross section of the vent-mast line as a criterion at a relative wind speed of $10 \mathrm{~m} / \mathrm{s}$ : Based on the arrangements of (a) reference; (b) 1-23-4; (c) 12-34; and (d) zigzag.

\subsection{Arrangement of Vent Masts}

Figure 6 shows the contour lines of the FL for each arrangement. The LFL merged when any of the two vent masts were close. Figure 7 compares the total volume of the FZ with four types of arrangements using the plot of the average volume. The fluctuation of the volume of the FZ $\left(V_{f z}\right)$ during iteration is plotted as the error bar. At a relative wind speed of $10 \mathrm{~m} / \mathrm{s}$, the reference arrangement records the lowest volumes of $7791 \mathrm{~m}^{3}$ and $4594 \mathrm{~m}^{3}$ for the 1-23-4 arrangement at a relative wind speed of $20 \mathrm{~m} / \mathrm{s}$. Figure 8 shows the area of the FZ at $5 \mathrm{~m}$ spacings in the drifting direction. The length of the FZ is defined from this plot as the length for which the area of the FZ becomes zero. This length is $220 \mathrm{~m}$ for the reference arrangement and $240 \mathrm{~m}$ for the 1-23-4 arrangement at a relative wind speed of $10 \mathrm{~m} / \mathrm{s}$; at $20 \mathrm{~m} / \mathrm{s}$, these lengths are 245 and $230 \mathrm{~m}$, respectively. In other words, the length increased for the reference arrangement, while the length decreased for the 1-23-4 arrangement as the relative wind speed increased from $10 \mathrm{~m} / \mathrm{s}$ to $20 \mathrm{~m} / \mathrm{s}$. Hence, the rank of volume changed dramatically for the 1-23-4 arrangement; it shows the highest rank at $10 \mathrm{~m} / \mathrm{s}$, but the lowest rank at $20 \mathrm{~m} / \mathrm{s}$ as in Figure 7. 


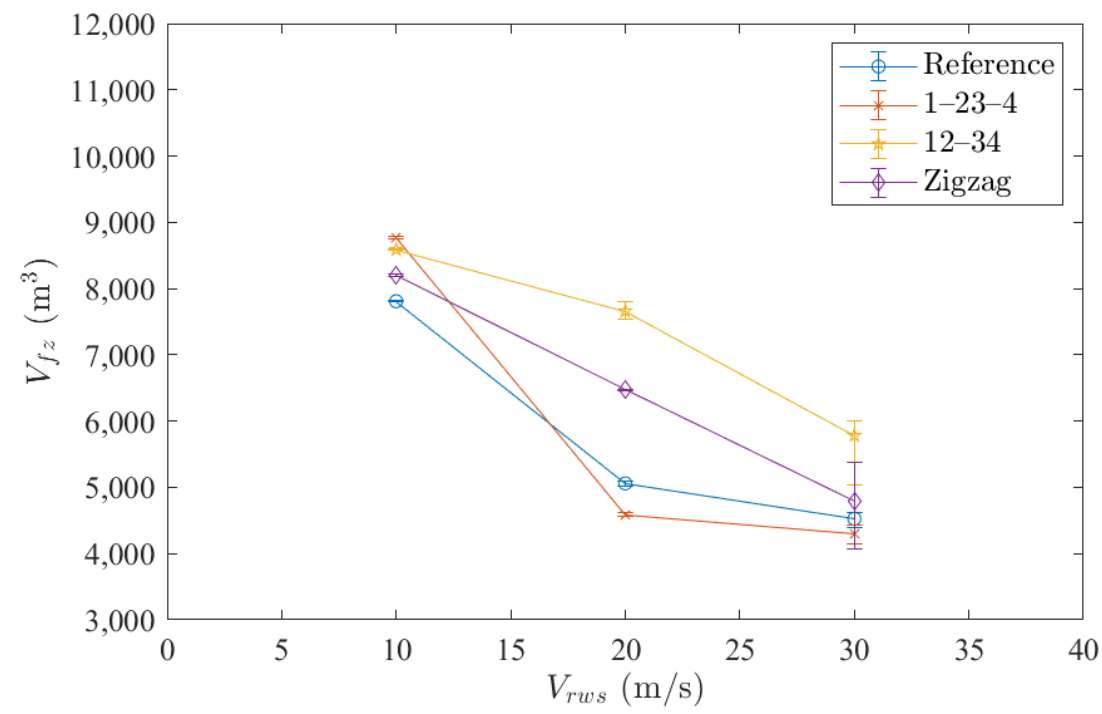

Figure 7. Total volume of the FZ $\left(V_{f z}\right)$ based on the relative wind speed $\left(V_{r w s}\right)$ for each arrangement of the vent masts.

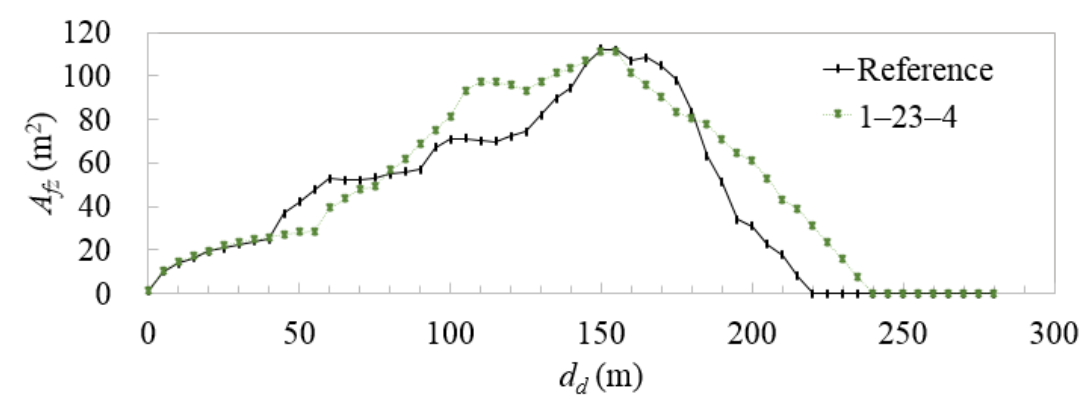

(a)

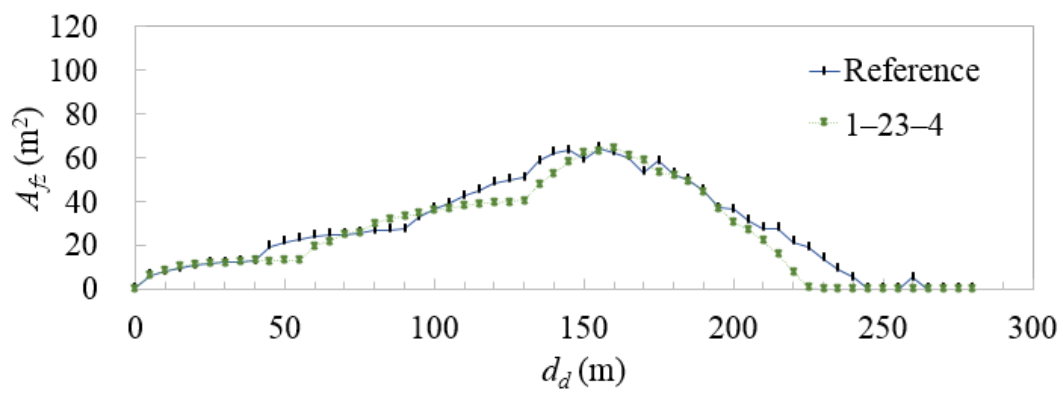

(b)

Figure 8. Area of FZ $\left(A_{f z}\right)$ at every $5 \mathrm{~m}$ cross section for the arrangement of 1-23-4 and reference based on the drifting distance $\left(d_{d}\right)$ from the first vent mast: at a relative wind speed of (a) $10 \mathrm{~m} / \mathrm{s}$, and (b) $20 \mathrm{~m} / \mathrm{s}$.

\subsection{Combination of Emission Patterns Selected among the Four Vent Masts}

Each of the four vent masts can emit hydrogen. Different emission scenarios are generated by selecting vents for emission from among the four available vent masts. Figure 9 compares the relative volume ratio of the scenarios. This is a ratio of the volumes of the FZs obtained using Equation (7). The denominator is the volume in an emission scenario where hydrogen is emitted from the first vent mast alone. The numerator is the volume for a specific scenario of emissions in which specific vents from among the four are selected. In the scenario that the second vent mast alone emits hydrogen, the differences were not greater than 3\% compared with the scenario in which hydrogen 
is emitted from the first vent mast alone. When a scenario of emission from two vents is considered, the volume increases greatly to $232 \%$, which exceeds $32 \%$ by $200 \%$. The volume ratio is significantly increased to over $400 \%$ for the scenarios of emissions from all the four vent masts, and it increases to at least $497 \%$ or up to $796 \%$ as the maximum values for relative wind speeds of 10 and $30 \mathrm{~m} / \mathrm{s}$, respectively. Two scenarios were used to identify the reason for these increases. In one scenario, emission occurs from the second vent mast alone, as shown in Figure 10a. In the other one, simultaneous emissions occur from the first and second vent masts, as shown in Figure 10b. The contour lines of the FL are plotted on the cross sections, as shown in Figure 10, to distinguish the lower region from the upper region of the FZ. The convection is shown by the fluid vectors. It is seen that convection acts on the FZ. The convection in the lower region of the FZ is weaker in the scenario of simultaneous emissions from the two vent masts compared with the scenario of emission from the second vent mast alone, as shown in Figure 10c,d. The convection - which dilutes the FZ by providing fresh air-acting on the lower region of the FZ is reduced for the scenario of simultaneous emissions. This reduction increases the volume of the FZ. Meanwhile, the upper region of the FZ rapidly floats with a strong uplifting of convection, as shown in Figure 10d where emissions are simultaneous for the two vent masts. This rapid floating allows the hydrogen to overlap with others, namely, the hydrogen emitted from the second vent mast overlaps with the hydrogen emitted from the first vent mast. This overlap broadens the FZ, as shown in Figure 10d. As a result, the volume of the $\mathrm{FZ}$ is $232 \%$.

\subsection{Direction of Emission from the Vent-Mast Outlet}

Three directions of emission are shown in Figure 4. Figure 11 compares the total volume of the FZ for these three directions. A scenario of emission wherein all four vent masts emit hydrogen is considered. The differences in the volume of the FZ for each direction of the vent mast outlet are shown in the figure. The volume is $7809 \mathrm{~m}^{3}$ for the upward and downward outlets and $7738 \mathrm{~m}^{3}$ for the downward outlet at a relative wind speed of $10 \mathrm{~m} / \mathrm{s}$. In other words, the volume of the FZ is lower in the downward direction than in the upward and downward directions of the outlet at a relative wind speed of $10 \mathrm{~m} / \mathrm{s}$.

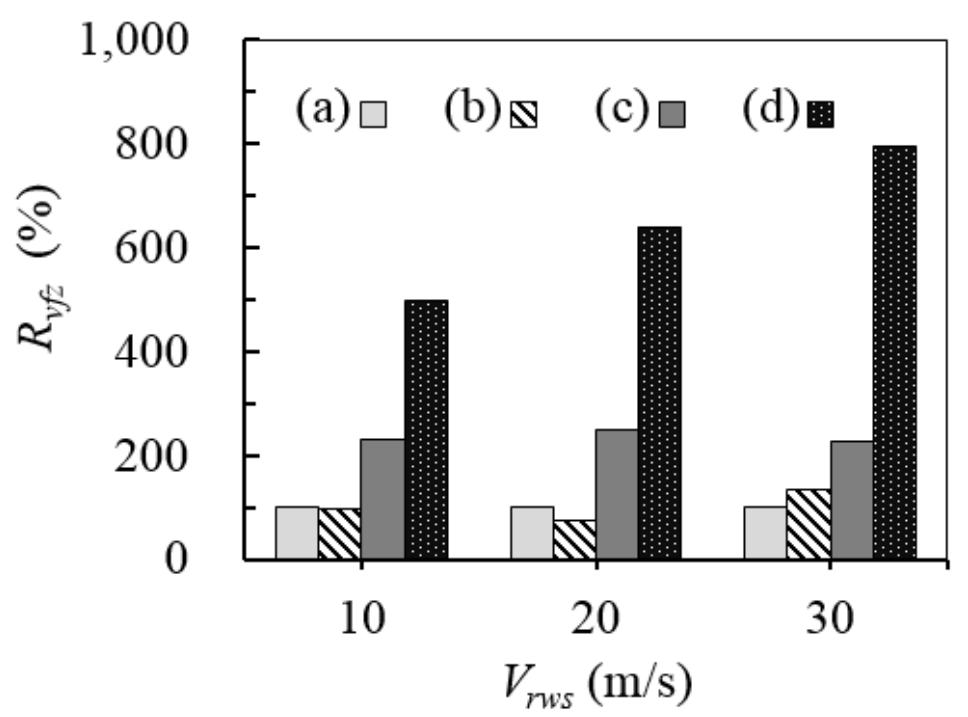

Figure 9. Relative volume ratio $\left(R_{v f z}\right)$ based on the relative wind speed $\left(V_{r w s}\right)$ for four scenarios, based on selection of emitting vent masts of the original type of outlet; (a) emission from the first vent mast alone; (b) emission from the second vent mast alone; (c) emissions from both of the first and second vent masts; and (d) emissions from all of the four vent masts. 


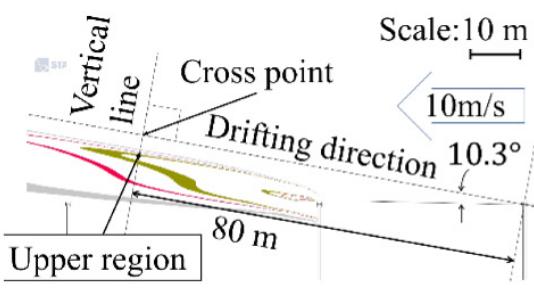

(a)

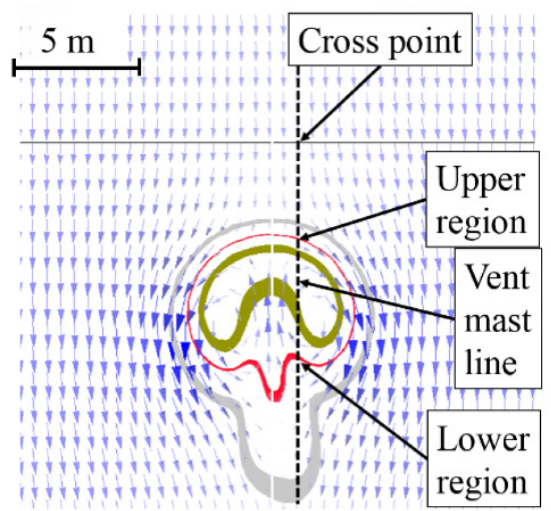

(c)

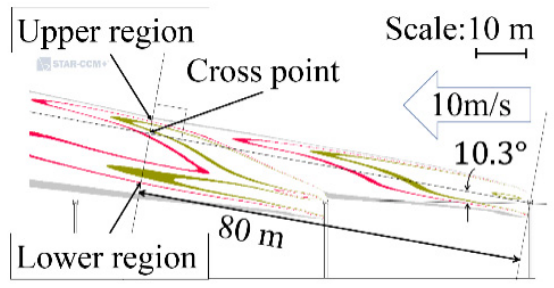

(b)

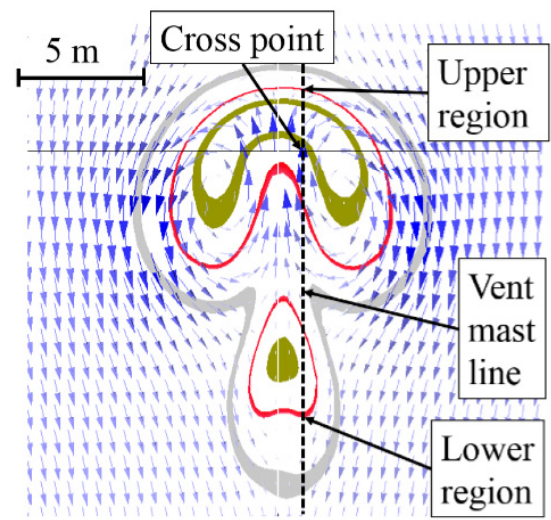

(d)

\begin{tabular}{|c|c|c|c|}
\hline & & & \\
\hline 0.0 & 0.6 & $\begin{array}{ll}1.2 & 1.8\end{array}$ & 2.4 \\
\hline
\end{tabular}

Figure 10. Contour lines of the FL with vectors at the cross section at $80 \mathrm{~m}$ drifting distance at a relative wind speed of $10 \mathrm{~m} / \mathrm{s}$. The left side of the scene $(\mathbf{a}, \mathbf{c})$ represents the scenario of emission from the second vent mast alone. The right side $(\mathbf{b}, \mathbf{d})$ represents the scenario of simultaneous emission from the first and second vent masts. The FL for the cross section of the vent-mast line in $(\mathbf{a}, \mathbf{b})$ and for the cross section of the vertical line $(\mathbf{c}, \mathbf{d})$ at a drifting distance of $80 \mathrm{~m}$ are shown.

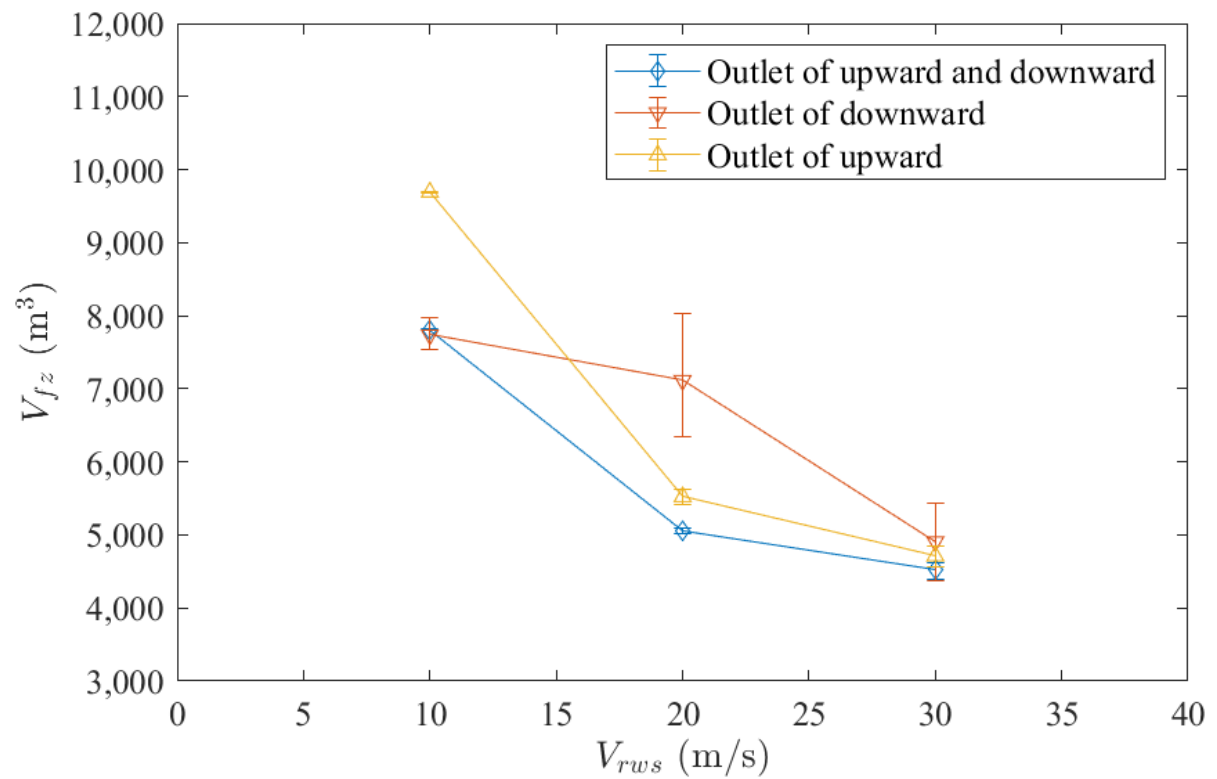

Figure 11. Total volume of FZ $\left(V_{f z}\right)$ for three kinds of vent-mast outlet directions based on relative wind speed $\left(V_{r w s}\right)$; the scenario of emissions from all of the four vent masts is shown. 
Additional scenarios of emission were created by using the first vent mast alone to identify the different actions of convection on the FZ for two types of outlet directions. The downward emission is compared with the upward and downward emissions to compare the length of the FZ. The length is defined by the drifting distance where the area of the FZ becomes zero, as mentioned in Section 3.2. The drifting distance starts from the center of the first vent mast outlet. It is $10.3^{\circ}$ in the clockwise direction from a parallel line of the DLWL, as shown in Figure 10. The area of the FZ is plotted for every $5 \mathrm{~m}$ of the cross sections for each scenario, as shown in Figure 12. The figure shows the differences in the lengths of the FZ. The length is longer for outlets with upward and downward emissions than for the scenario with the downward emission only. The length for upward and downward emissions is $160 \mathrm{~m}$, and that for only downward emission is $150 \mathrm{~m}$.

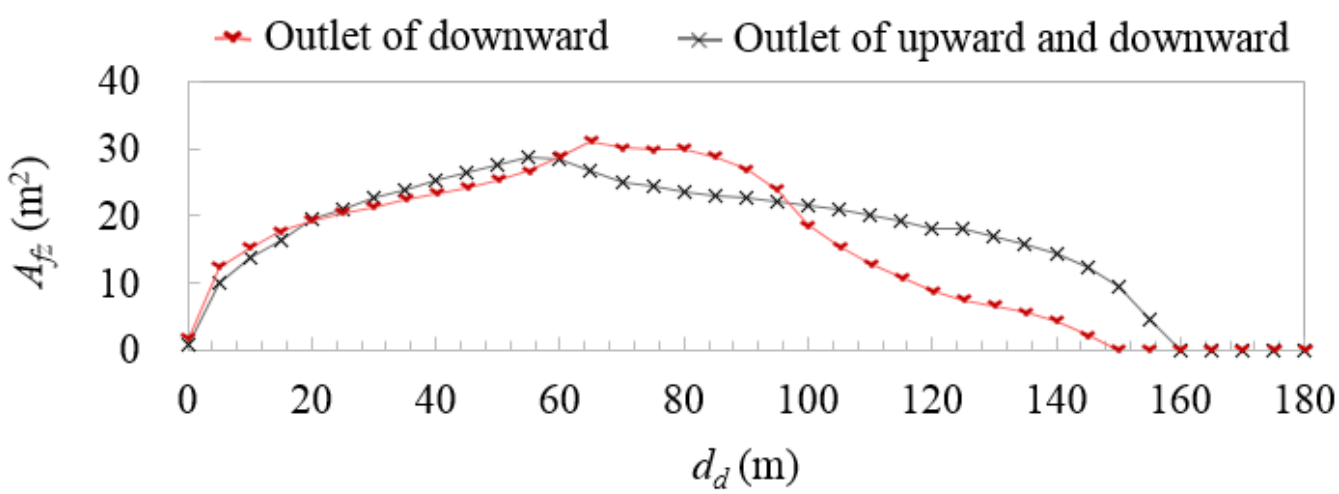

Figure 12. Area of FZ $\left(A_{f z}\right)$ for the cross section of vertical line at every $5 \mathrm{~m}$ drifting distance $\left(d_{d}\right)$; the scenario of emission from the first vent mast alone at a relative wind speed of $10 \mathrm{~m} / \mathrm{s}$ is shown.

Further, a quantitative method was used to compare the flammable areas of the two outlet types. The summation of the flammable area is defined as an integrated volume. The integrated volume is the approximate integration of the area of the FZ $\left(A_{f z}\right)$ based on the drifting distance $\left(d_{d}\right)$. It is estimated using the trapezoidal rule [29]. The angle of the drifting direction from the DLWL differs between the scenarios of the different outlet directions. The angle is $10.3^{\circ}$ for the outlet for upward and downward emission and $4.9^{\circ}$ for the outlet for the outlet for downward emission. The integrated volume for the outlet for upward and downward emission is $2206 \mathrm{~m}^{3}$ up to a drifting distance of $100 \mathrm{~m}$ and $2339 \mathrm{~m}^{3}$ for the outlet for downward emission. The volume emitted in the downward direction is more than that emitted in both directions only by $6 \%$. Up to $110 \mathrm{~m}$, the outlet for downwards emission showed an increase of 3\% compared to the outlet for emission in both directions. This is a decrease of 3\% from the integration up to a distance of $100 \mathrm{~m}$. This decrease is originated from a different location of the center of the vortex inside the FZ. Cross sections of the vertical line at drifting distances of 100 and $110 \mathrm{~m}$ for each scenario are shown in Figure 13. This figure shows not only the contour lines of the FL, but also the tangential velocity vectors. The center of the vortex is inside the FZ in the scenario of emission in both directions from the outlet, while it is located outside the FZ in the scenario of downward emission. It is expected that the vortex dissipates the FZ more efficiently when the center of the vortex is located outside, as it brings more fresh air into the FZ. 


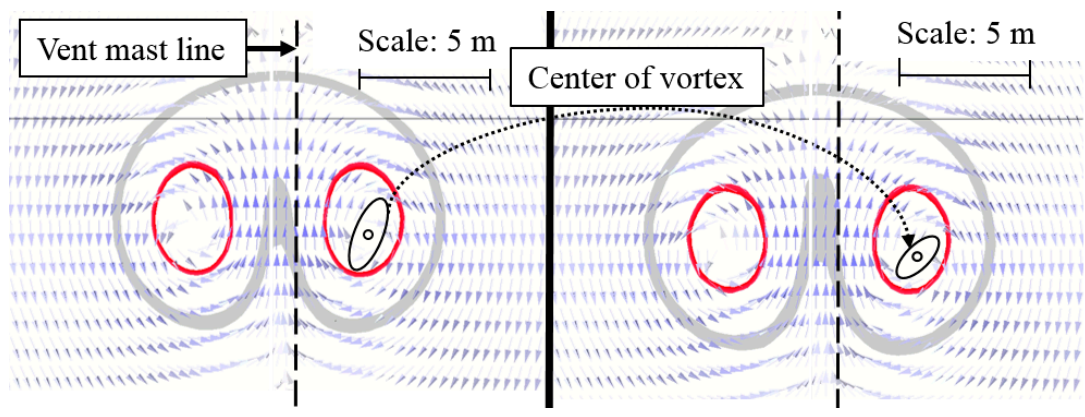

(a)

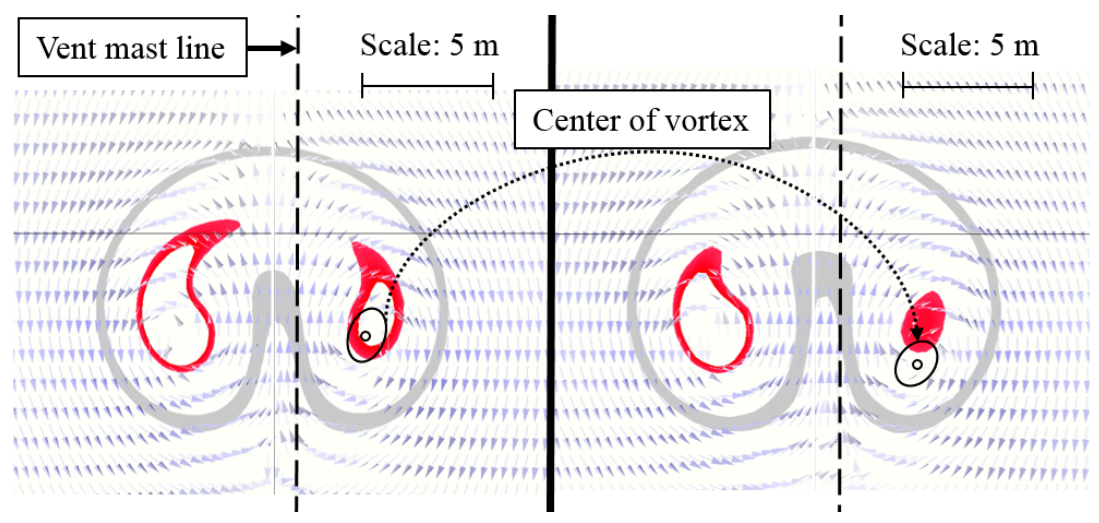

(b)

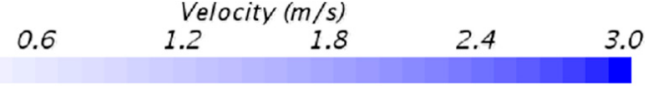

Figure 13. Contour lines of the FL and the tangential velocity of fluid. The left side of the figure shows the cross section at a drifting distance of $100 \mathrm{~m}$; the right side of the figure represents the results for a drifting distance of $110 \mathrm{~m}$. Different directions of vent-mast outlets for scenarios are shown: (a) is for the outlet with both upward and downward emission, while (b) is for the outlet with downward emission. The relative wind speed is $10 \mathrm{~m} / \mathrm{s}$ for both scenarios.

\section{Conclusions}

In the present study, four parameters that affect the volume and length of an FZ were examined. These parameters are the relative wind speed, arrangement of vent masts, combination of the emitting vent masts, and direction of emission from the outlet of vent mast. Various scenarios were built with the four parameters. The volume and length of the FZ for each scenario were examined. The results showed that convection contributes to the differences between the scenarios. Convection dissipates the FZ by bringing in fresh air. Therefore, a reduction in convection prevents the dissipation of the FZ, and this may result in a significant increase of volume of the FZ. However, this increase cannot be explained only by the reduction in convection. A strong convection can also broaden the FZ by supplying it with hydrogen from a nearby FZ, and the location of the vortex affects the length of the FZ. The FZ is elongated by the trapping of hydrogen via recirculation inside the FZ. On the other hand, the FZ may be shortened by the vortex providing fresh air into the FZ if the center of the vortex is located outside the FZ.

Author Contributions: Conceptualization, H.L., B.C.C. and G.R.; methodology, G.W.K.; software, H.N.; validation, H.N., G.W.K. and Y.N.; formal analysis, H.N.; investigation, H.N.; resources, H.N. and H.L.; data curation, H.N. and H.L.; writing—original draft preparation, H.N.; writing-review and editing, G.W.K. and Y.N.; visualization, H.N.; supervision, G.W.K. and Y.N.; project administration, Y.N.; funding acquisition, Y.N. All authors have read and agreed to the published version of the manuscript. 
Funding: This research was supported by the National R\&D Program through the National Research Foundation of Korea (NRF) funded by the Ministry of Science, ICT and Future Planning (grant number NRF-2019M3E6A1064703 and 2020M1A2A2080880) and the Korea Evaluation Institute of Industrial Technology (KEIT) funded by the Ministry of Trade, Industry and Energy (grant number 20009835).

Institutional Review Board Statement: Not applicable.

Informed Consent Statement: Not applicable.

Conflicts of Interest: The authors declare no conflict of interest.

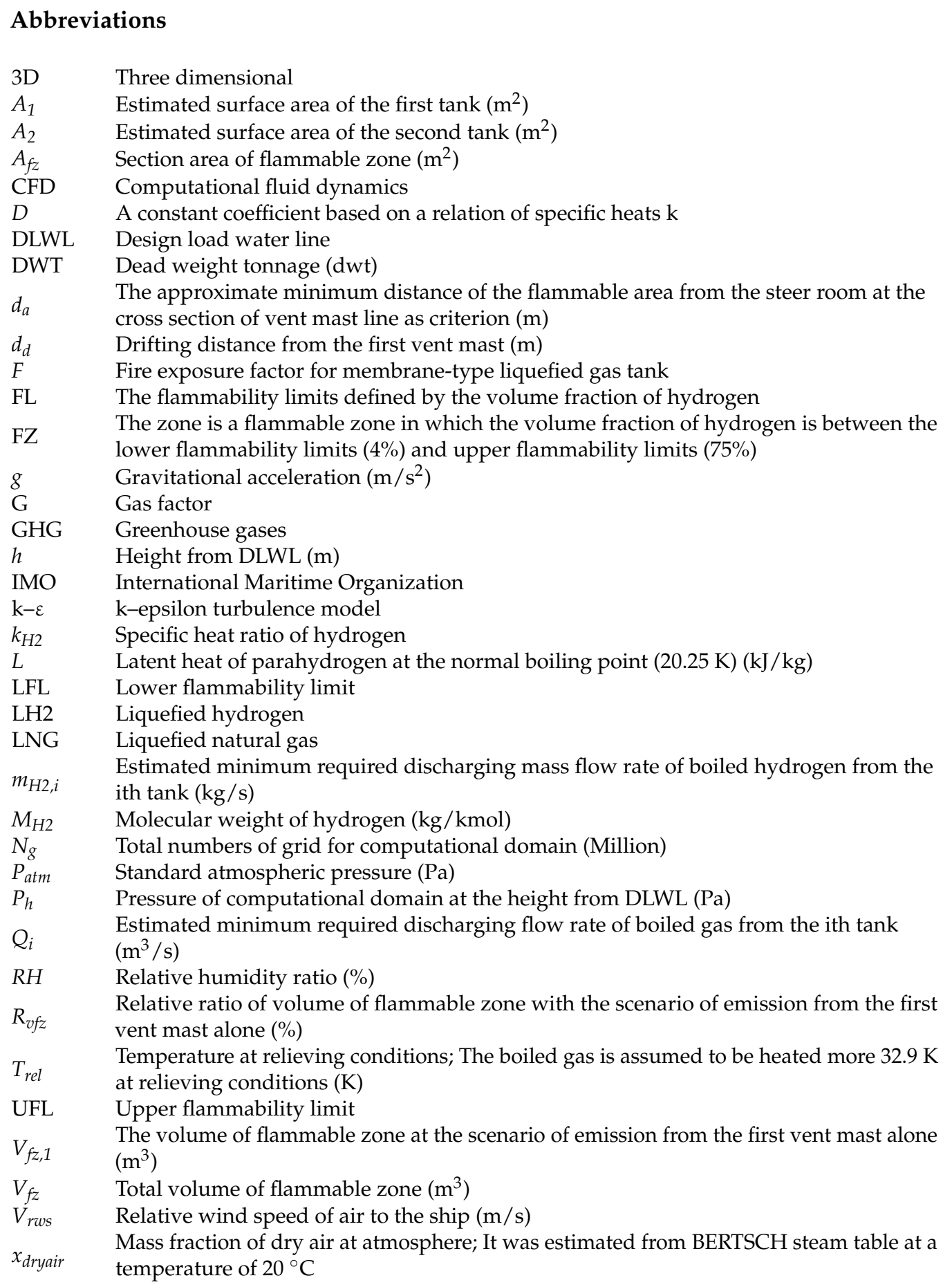




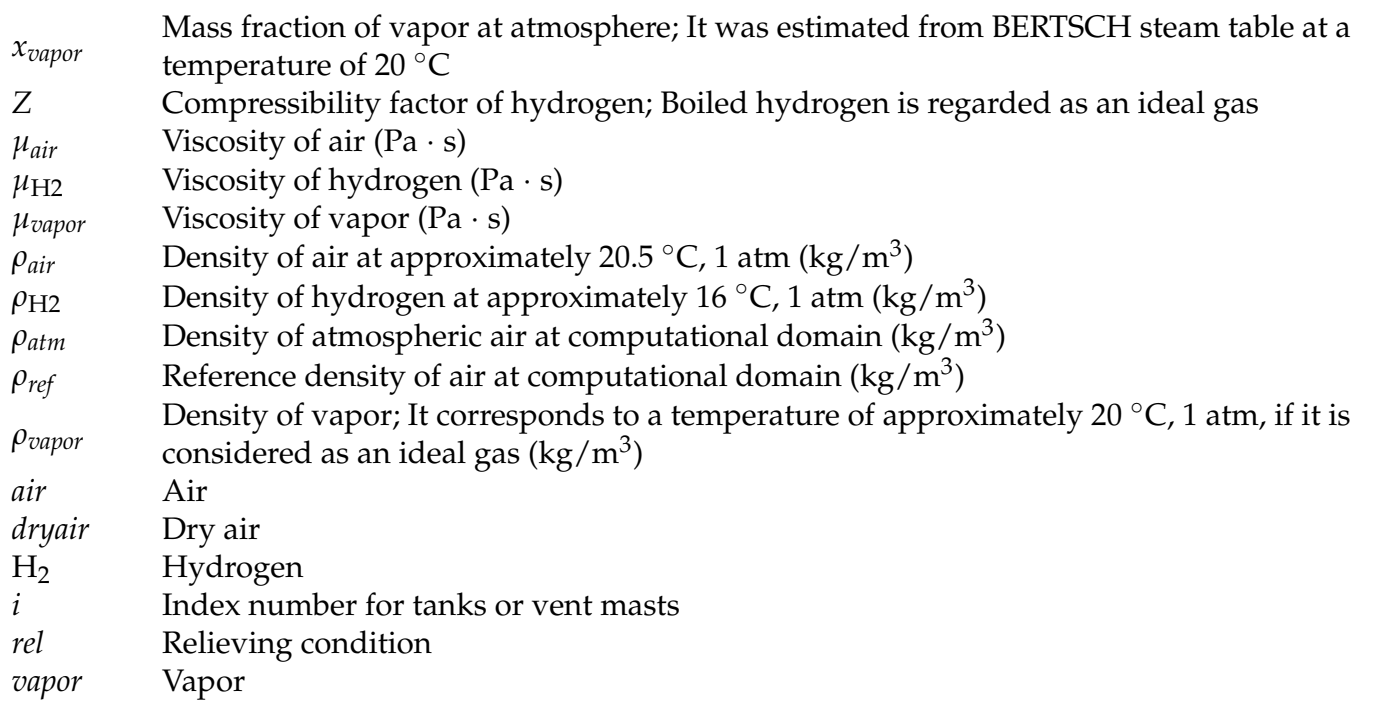

\section{References}

1. Pulgar-Vida, M. Report of the Conference of the Parties on Its Twenty-First Session, Held in Paris from 30 November to 13 December 2015: Addendum. Part Two: Action Taken by the Conference of the Parties at Its Twenty-First Session; United Nations Framework Convention on Climate Change Bonn: Bonn, Germany, 2015.

2. Saito, H. Initial IMO Strategy on Reduction of GHG Emissions from Ships; Resolution MEPC.304(72); IMO: London, UK, 2018.

3. Adamchak, F.; Adede, A. LNG as marine fuel. In Proceedings of the 17th International Conference and Exhibition of Liquefied Natural Gas, Houston, TX, USA, 16-19 April 2013.

4. Olmer, N.; Comer, B.; Roy, B.; Mao, X.; Rutherford, D. Greenhouse Gas Emissions from Global Shipping, 2013-2015 Detailed Methodology International Council on Clean Transportation: Washington, DC, USA, 2017; pp. 1-38.

5. Breinholt, C. Adoption of the International Code of Safety for Ships Using Gases or Other Low-Flashpoint Fuels (IGF Code); Resolution MSC.391(95); IMO: London, UK, 2015.

6. Markowski, J.; Pielecha, I. The potential of fuel cells as a drive source of maritime transport. IOP Conf. Ser. Earth Environ. Sci. 2019, 214, 012019. [CrossRef]

7. New Energy and Industrial Technology Development Organization (NEDO). Development of Technologies for Realizing Hydrogen Society. Available online: https://www.nedo.go.jp/english/activities/activities_ZZJP_100096.html (accessed on 14 November 2021).

8. $\mathrm{CO}_{2}$-Free Hydrogen Energy Supply-Chain technology Research Association (HyStra), Hydrogen Energy Supply Chain Pilot Project between Australia and Japan. Available online: http:/ / www.hystra.or.jp/en/project/ (accessed on 14 November 2021).

9. Lee, H.; Shao, Y.; Lee, S.; Roh, G.; Chun, K.; Kang, H. Analysis and assessment of partial re-liquefaction system for liquefied hydrogen tankers using liquefied natural gas (LNG) and H2 hybrid propulsion. Int. J. Hydrog. Energy 2019, 44, 15056-15071. [CrossRef]

10. International Maritime Organization. International Code for the Construction and Equipment of Ships Carrying Liquefied Gases in Bulk (IGC Code); IMO Publishing: London, UK, 2016; pp. 103-108.

11. Roh, G.; Na, Y.; Park, J.-Y.; Kim, H. Analysis of Internal Gas Leaks in an MCFC System Package for an LNG-Fueled Ship. Appl. Sci. 2019, 9, 2330. [CrossRef]

12. Kim, J.-H.; Doh, D.-H.; Choi, B.C. Evaluation of the ventilation safety requirements for the fuel gas supply system room of a gas-fueled vessel: Simulated leaks of methane and propane. J. Mech. Sci. Technol. 2018, 32, 5521-5532. [CrossRef]

13. Doh, D.-H.; Chun, K.W.; Ninomiya, N.; Choi, B.C. A swirl static mixer with diluent for reducing the flammable extent of venting gases in a low-flashpoint fueled vessel. J. Mech. Sci. Technol. 2019, 33, 3311-3321. [CrossRef]

14. Blaylock, M.; Pratt, J.; Bran-Anleau, G.; Proctor, C. Informing Hazardous Zones for On-Board Maritime Hydrogen Liquid and Gas Systems; Sandia National Lab. (SNL-NM): Albuquerque, NM, USA, 2018.

15. Kang, H.-K. An examination on the dispersion characteristics of boil-off gas in vent mast exit of membrane type LNG carriers. J. Korean Soc. Mar. Environ. Saf. 2013, 19, 225-231. [CrossRef]

16. Hansen, O.R. Liquid hydrogen releases show dense gas behavior. Int. J. Hydrog. Energy 2020, 45, 1343-1358. [CrossRef]

17. Cao, Y.; Jia, Q.-J.; Wang, S.-M.; Jiang, Y.; Bai, Y. Safety design analysis of a vent mast on an LNG powered ship during a low temperature combustible gas leakage accident. J. Ocean Eng. Sci. 2021. [CrossRef]

18. Cornwell, J.B.; Johnson, D.W.; Martinsen, W.E. Relief Valves and Vents: How Exit Conditions Affect Hazard Zones. In Proceedings of the American Institute of Chemical Engineers 1990 Summer National Meeting, San Diego, CA, USA, 19-22 August 1990.

19. Molland, A.F. The Maritime Engineering Reference Book: A Guide to Ship Design; Butterworth-Heinemann: Oxford, UK, 2008; pp. 52-56.

20. Class, N.K. Guidelines for Liquefied Hydrogen Carriers; Class NK: Tokyo, Japan, 2017. 
21. White, F.M. Fluid Mechanics, in SI Units; McGraw-Hill: New York, NY, USA, 2011; p. 840.

22. Flynn, T. Cryogenic Engineering, Revised and Expanded, 2nd ed.; Marcel Dekker: New York, NY, USA, 2008; pp. 185-186; 199; 201-204; 789.

23. Threlkeld, J.L. Thermal Environmental Engineering; Prentice Hall: Hoboken, NJ, USA, 1998; Appendix A, Table A.6SI.

24. Josef BERTSCH Ges. m.b.H \& Co. Steam Table. Available online: https://www.bertsch.at (accessed on 15 October 2020).

25. Hydrogen. Available online: https://pubchem.ncbi.nlm.nih.gov/compound/Hydrogen (accessed on 18 June 2019).

26. Standard Atmosphere. Available online: https://en.wikipedia.org/wiki/Standard_atmosphere_(unit) (accessed on 11 May 2021).

27. Beaufort Wind Force Scale. Available online: https://www.metoffice.gov.uk/weather/guides/coast-and-sea/beaufort-scale (accessed on 22 January 2021).

28. Schefer, R.; Evans, G.; Zhang, J.; Ruggles, A.; Greif, R. Ignitability limits for combustion of unintended hydrogen releases: Experimental and theoretical results. Int. J. Hydrog. Energy 2011, 36, 2426-2435. [CrossRef]

29. Griffiths, D.V.; Smith, I.M. Numerical Methods for Engineers, 7th ed.; McGraw-Hill Education: New York, NY, USA, 2016; pp. 475-481. 